\title{
Isotope effects in aqueous solvation of simple halides
}

\author{
Pablo E. Videla, ${ }^{1, a)}$ Peter J. Rossky, ${ }^{2}$ and D. Laria ${ }^{1,3, b)}$ \\ ${ }^{1}$ Departamento de Química Inorgánica Analítica y Química-Física e INQUIMAE, Facultad de Ciencias Exactas \\ y Naturales, Universidad de Buenos Aires, Ciudad Universitaria, Pabellón II, 1428 Buenos Aires, Argentina \\ ${ }^{2}$ Department of Chemistry, Rice University, Houston, Texas 77251-1892, USA \\ ${ }^{3}$ Departamento de Física de la Materia Condensada, Comisión Nacional de Energía Atómica, Avenida Libertador \\ 8250, 1429 Buenos Aires, Argentina
}

(Received 2 June 2017; accepted 11 August 2017; published online 6 September 2017)

\begin{abstract}
We present a path-integral-molecular-dynamics study of the thermodynamic stabilities of $\mathrm{DOH} \cdots \mathrm{X}^{-}$ and $\mathrm{HOD} \cdots \mathrm{X}^{-}(\mathrm{X}=\mathrm{F}, \mathrm{Cl}, \mathrm{Br}, \mathrm{I})$ coordination in aqueous solutions at ambient conditions. In agreement with experimental evidence, our results for the $\mathrm{F}^{-}$case reveal a clear stabilization of the latter motif, whereas, in the rest of the halogen series, the former articulation prevails. The $\mathrm{DOH} \cdots \mathrm{X}^{-}$preference becomes more marked the larger the size of the ionic solute. A physical interpretation of these tendencies is provided in terms of an analysis of the global quantum kinetic energies of the light atoms and their geometrical decomposition. The stabilization of the alternative ionic coordination geometries is the result of a delicate balance arising from quantum spatial dispersions along parallel and perpendicular directions with respect to the relevant $\mathrm{O}-\mathrm{H} \cdots \mathrm{X}^{-}$axis, as the strength of the waterhalide $\mathrm{H}$-bond varies. This interpretation is corroborated by a complementary analysis performed on the different spectroscopic signals of the corresponding IR spectra. Published by AIP Publishing. [http://dx.doi.org/10.1063/1.4986231]
\end{abstract}

\section{INTRODUCTION}

Ionic hydration is a fundamental element of solution chemistry. For the specific case of simple anionic species, the $\mathrm{OH} \cdots \mathrm{X}^{-}(\mathrm{X}=\mathrm{F}, \mathrm{Cl}, \mathrm{Br}, \mathrm{I}), \mathrm{H}$-bonded, coordination pattern is the molecular "fingerprint" of the phenomenon, prevailing in condensed phases, clusters, and in the simplest $\mathrm{X}^{-} \cdot\left[\mathrm{H}_{2} \mathrm{O}\right]$ dimer case. ${ }^{1}$ The H-bonded geometry contrasts with the alternative, ion-dipole alignment, in which the two aqueous hydrogens would equally participate in the ion-water binding, in a symmetrical structure. That dipolar geometry is valid at large separations. Interestingly, in bulk phases, the $\mathrm{H}$-bond articulation minimizes disruption in the three dimensional intermolecular architecture within the solvent, by facilitating water-water (W-W) H-bonds, connecting the first to the second solvation shells of the anions. This tendency underscores the competitive characteristics of solute-water and $\mathrm{W}-\mathrm{W}$ interactions that are of interest here.

For more than thirty years, ${ }^{2,3}$ there has been a sustained interest in assessing the actual extent of the disruptions of the structure in the neat solvent promoted by the presence of the solute ionic Coulomb field. As such, the concepts of "structure makers" and "structure breakers" have been coined to refer to solutes that generate augmentation or depletion in the H-bonding among water molecules in the solvation shells of solutes. ${ }^{4}$ Recently, the molecular structural and dynamical implications of these phenomenological concepts have been contested, and there are important

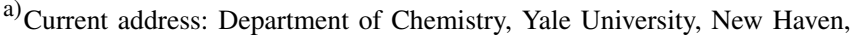
CT 06520-8107, USA.

b) Author to whom correspondence should be addressed: dhlaria@cnea.gov.ar
}

characteristics that still await proper clarifications. ${ }^{5-8}$ This is in large part due to limitations in the interpretation of direct spectral signals, which can stem from a variety of $\mathrm{H}$-bonding scenarios. ${ }^{9,10}$

The present analysis represents a natural continuation of two recent studies, in which we examined isotopic stabilities along H-bonds, in the vicinity of hydrogen halides and $\mathrm{I}^{-}$dissolved in aqueous nanoclusters that combine $\mathrm{HOD}$ and $\mathrm{H}_{2} \mathrm{O}$, at temperatures close to $\sim 50 \mathrm{~K} .{ }^{11,12}$ The results that emerged from these studies revealed a variety of isotopic preferences, which are the result of delicate interplays between inter and intramolecular couplings, which, in turn, are controlled by the topology of the corresponding three dimensional potential energy surfaces. As such, in what follows, we will present a comprehensive description of characteristics of H-bonding in the first solvation shells of several simple halides in solution, from a molecular perspective that focuses on preferential solvation by different isotopic species. Our motivation here focuses on assessing the validity of the generalities of our previous conclusions in more conventional, bulk phases at ambient conditions, where quantum effects should manifest in a more subtle fashion. Our analysis will rely on path-integral molecular dynamics simulation results that shed light on the basic elements that control the relative stabilities of $\mathrm{OH} \cdots \mathrm{X}^{-}$ and $\mathrm{OD} \cdots \mathrm{X}^{-}$motifs in bulk environments at ambient conditions. In a broader context, the present study presents some common elements with a previous one ${ }^{13}$ performed on rigid water that focused on the effects of quantum fluctuations on the $\mathrm{O}-\mathrm{X}^{-}$potential of mean force, the reorientation dynamics of the ionic first solvation shell and the low frequency branch of the IR spectrum.

The organization of the present work is as follows: In Sec. II we present the model and technical details about the 
simulation procedure. The main results for the work are presented in Sec. III. In Sec. IV we summarize the most important conclusions of the paper.

\section{MODEL AND SIMULATION PROCEDURE}

The systems under investigation were models of infinite dilute $\mathrm{X}^{-}$halides $(\mathrm{X}=\mathrm{F}, \mathrm{Cl}, \mathrm{Br}, \mathrm{I})$, dissolved in aqueous phases comprising 215 molecules, confined in periodically replicated, cubic boxes of length $L=18.64 \AA$. Our simulation approach relied on the consideration of the following $P$-bead representation of the path-integral canonical partition function for an $N$ particle system, at a temperature $T,{ }^{14}$

$$
Q_{P}=\frac{1}{h^{3 P N}} \int \cdots \int \prod_{k=1}^{P} \prod_{i=1}^{N} \mathrm{~d} \mathbf{r}_{i}^{(k)} \mathrm{d} \mathbf{p}_{i}^{(k)} e^{-\beta_{P} H_{P}\left(\left\{\mathbf{p}_{i}^{(k)}\right\},\left\{\mathbf{r}_{i}^{(k)}\right\}\right)},
$$

where $\left(P \beta_{P}\right)^{-1}=k_{B} T$ and

$$
\begin{aligned}
H_{P}\left(\left\{\mathbf{p}_{i}^{(k)}\right\},\left\{\mathbf{r}_{i}^{(k)}\right\}\right)= & \sum_{i=1}^{N} \sum_{k=1}^{P}\left[\frac{\left(\mathbf{p}_{i}^{(k)}\right)^{2}}{2 M_{i}}+\frac{M_{i} \omega^{2}}{2}\left(\mathbf{r}_{i}^{(k)}-\mathbf{r}_{i}^{(k+1)}\right)^{2}\right] \\
& +\sum_{k=1}^{P} V\left(\mathbf{r}_{1}^{(k)}, \mathbf{r}_{2}^{(k)}, \ldots, \mathbf{r}_{N}^{(k)}\right)
\end{aligned}
$$

in the previous equation, $\omega=\left(\beta_{P} \hbar\right)^{-1}$, whereas $\mathbf{r}_{i}^{(k)}$ and $\mathbf{p}_{i}^{(k)}$ represent the position and momentum of the $i$ th particle of mass $M_{i}$ at the imaginary time slice $k$, respectively $\left(\mathbf{r}_{i}^{(P+1)}=\mathbf{r}_{i}^{(1)}\right) . V$ represents the potential energy function of the system.

Thermal averages for position dependent observables $\mathcal{O}(\{\mathbf{r}\})$ are expressed as

$$
\langle\mathcal{O}\rangle_{P}=\frac{1}{Q_{P} h^{3 P N}} \int \cdots \int \prod_{k=1}^{P} \prod_{i=1}^{N} \mathrm{~d} \mathbf{r}_{i}^{(k)} \mathrm{d} \mathbf{p}_{i}^{(k)} e^{-\beta_{P} H_{P}} \mathcal{O}_{P}\left(\left\{\mathbf{r}_{i}^{P}\right\}\right)
$$

with

$$
\mathcal{O}_{P}\left(\left\{\mathbf{r}_{i}^{P}\right\}\right)=\frac{1}{P} \sum_{k=1}^{P} \mathcal{O}\left(\left\{\mathbf{r}_{i}^{(k)}\right\}\right) .
$$

Equilibrium ensemble averages were computed from $\sim 1 \mathrm{~ns}$, canonical trajectories, generated by introducing a transformation from Cartesian to normal-mode coordinates, ${ }^{15} \mathrm{cou}-$ pled to a multiple time step algorithm ${ }^{16}$ that discriminates fast, nearly harmonic, intramolecular interactions in the quantum polymers and intramolecular contributions to $V$, from the rest of the slowly varying components of the forces $(\delta t$ $=\Delta t / 4, \Delta t=0.1 \mathrm{fs}$ ). Appropriate temperature control (within $0.1 \mathrm{~K}$ ) was obtained by implementing an efficient local path integral Langevin thermostat set at $T=298 \mathrm{~K}$, with targeted optimal sampling on the internal ring-polymer normal modes and a white noise Langevin thermostat applied to the centroid, with a time constant of 1 ps. ${ }^{17}$ In this thermal regime, we obtained adequate convergence of the results by setting $P$ to 32 . The long range nature of Coulomb interactions were treated by implementing Ewald sums, using a particle-mesh algorithm. $^{18}$

The potential energy term $V(\{\mathbf{r}\})$ in Eq. (2) included water-water and solute-water contributions, namely,

$$
V(\{\mathbf{r}\})=V_{w w}(\{\mathbf{r}\})+V_{s w}(\{\mathbf{r}\}) .
$$

Following our previous studies, for $V_{w w}(\{\mathbf{r}\})$, we adopted the 4-site, nonharmonic, q-TIP4P/F model, ${ }^{19}$ originally based on the TIP4P/2005 parametrization. ${ }^{20}$ The former model has shown to provide reasonable estimates for isotopic exchange equilibria in a variety of media, including bulk water at ambient conditions. ${ }^{21,22}$ Ion-water parameters were taken from Ref. 23 for the related, 4-site, TIP4P model, without further modifications. In the present case, we made no efforts to explore possible effects derived from the absence of polarization contributions to the effective ion-water interactions. As an illustrative test of the performance of this combination of force fields, in Fig. 1, we present classical results for ionoxygen radial distribution function for all ions investigated. All profiles present reasonable agreement with previous classical simulations. $^{23-28}$

Hydrogen bonding was defined in terms of a geometrical criterion. ${ }^{13,29,30}$ In particular, a water molecule was considered $\mathrm{H}$-bonded to a given $\mathrm{X}^{-}$solute, provided the oxygenhalide distance falls within the first ionic solvation shell (i.e., $\left.r_{\mathrm{OX}^{-}} \leq r_{\text {min }}\right)$ and the $\mathrm{H}-\mathrm{O}-\mathrm{X}^{-}$angle was $\theta_{\mathrm{HOX}^{-}} \leq 30^{\circ}$. Similar criteria define water-water $\mathrm{H}$-bond connectivities, except that, for these cases, the maximum threshold $\mathrm{O}-\mathrm{O}$ distance was set to $r=3.5 \AA$. The values of $r_{\text {min }}$ were obtained from the outer boundary of the ion first solvation shell, based on the radial distribution functions, as displayed in Fig. 1.

Temporal correlations were computed assuming the Ring Polymer Molecular Dynamics approximation for Kubotransformed time correlation functions, namely, ${ }^{31,32}$

$$
\begin{aligned}
K_{\mathcal{A B}}(t)= & \frac{1}{\beta Q} \int_{0}^{\beta} \mathrm{d} \lambda \operatorname{Tr} e^{-\beta \hat{H}} \\
& \times \hat{\mathcal{A}}(-i \hbar \lambda) \hat{\mathcal{B}}(t) \sim\langle\mathcal{A}(0) \mathcal{B}(t)\rangle_{\mathrm{RPMD}},
\end{aligned}
$$

where $\hat{\mathcal{O}}(t)$ represents the Heisenberg representation of an operator $\hat{\mathcal{O}}$ evaluated at time $t$ and

$$
\begin{aligned}
\langle\mathcal{A}(0) \mathcal{B}(t)\rangle_{\mathrm{RPMD}}= & \frac{1}{Q_{P} h^{3 P N}} \int \ldots \int \prod_{k=1}^{P} \prod_{i=1}^{N} \mathrm{~d} \mathbf{r}_{i}^{(k)} \mathrm{d} \mathbf{p}_{i}^{(k)} \\
& \times e^{-\beta_{P} H_{P}} \mathcal{A}_{P}\left(\left\{\mathbf{r}_{i}^{P}(0)\right\}\right) \mathcal{B}_{P}\left(\left\{\mathbf{r}_{i}^{P}(t)\right\}\right) .
\end{aligned}
$$

In the previous equation, the time evolution of the set of coordinates $\left\{\mathbf{r}_{i}^{P}(t)\right\}$ is dictated by the classical equations of motion derived from the Hamiltonian appearing in Eq. (2), at a temperature $\left(k_{B} \beta_{P}\right)^{-1}$.

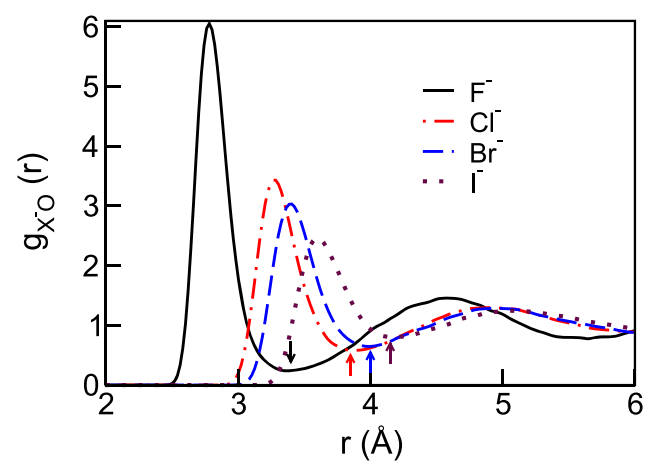

FIG. 1. Ion-Oxygen radial distribution functions for aqueous solutions at ambient conditions. The arrows indicate the threshold $\mathrm{O}-\mathrm{X}^{-}$distances for H-bond definition $\left(r_{\min }\right.$, see text). 


\section{RESULTS}

We will start our analysis by examining the following model reaction in water solution

$$
\mathrm{D}_{\mathrm{W}} \mathrm{OH}_{\mathrm{X}} \cdots \mathrm{X}^{-} \rightarrow \mathrm{H}_{\mathrm{W}} \mathrm{OD}_{\mathrm{X}} \cdots \mathrm{X}^{-},
$$

where the subscript $\mathrm{W}(\mathrm{X})$ denotes a site acting as $\mathrm{H}$-bond donor to a water $(\mathrm{W})$ molecule or $\mathrm{X}^{-}$species $(\mathrm{X}=\mathrm{F}, \mathrm{Cl}$, $\mathrm{Br}, \mathrm{I})$.

Preferential isotopic solvation is normally expressed in terms of population ratios $\alpha=e^{-\beta \Delta A}$, where $\Delta A$ is the free energy difference associated with the interchange shown in Eq. (8). In computer simulations, estimates for $\alpha$ can be readily obtained by implementing a scheme developed by Cheng and Ceriotti, ${ }^{33}$ based on the consideration of the following scaledcoordinate estimator, namely,

$$
\alpha=\frac{\left\langle\mathcal{Z}_{m_{\mathrm{H}}, m_{\mathrm{D}}}\right\rangle_{\mathrm{X}, m_{\mathrm{H}}}}{\left\langle\mathcal{Z}_{m_{\mathrm{H}}, m_{\mathrm{D}}}\right\rangle_{\mathrm{W}, m_{\mathrm{H}}}},
$$

where

$$
\begin{aligned}
\mathcal{Z}_{m_{\mathrm{H}}, m_{\mathrm{D}}}= & \exp \left[-\frac{\beta}{P} \sum_{k=1}^{P} V\left(\tilde{\mathbf{r}}_{1}^{(k)}, \mathbf{r}_{2}^{(k)}, \ldots, \mathbf{r}_{N}^{(k)}\right)\right. \\
& \left.-V\left(\mathbf{r}_{1}^{(k)}, \mathbf{r}_{2}^{(k)}, \ldots, \mathbf{r}_{N}^{(k)}\right)\right]
\end{aligned}
$$

In Eq. (10), $V\left(\mathbf{r}_{1}^{(k)}, \mathbf{r}_{2}^{(k)} \ldots, \mathbf{r}_{N}^{(k)}\right)$ represents the potential energy of the system at the $k$-th imaginary time slice, evaluated with Cartesian coordinates of the particles fixed at $\left\{\mathbf{r}_{N}^{(k)}\right\}$. Similarly, $V\left(\tilde{\mathbf{r}}_{1}^{(k)}, \mathbf{r}_{2}^{(k)}, \ldots, \mathbf{r}_{N}^{(k)}\right)$ represents the potential energy evaluated at the same set of coordinates, with the exception of the site undergoing $m_{\mathrm{H}} \rightarrow m_{\mathrm{D}}$ mass transformation, here arbitrarily labeled as particle 1 . The position of this tagged particle is rescaled according to

$$
\tilde{\mathbf{r}}_{1}^{(k)}=\overline{\mathbf{r}}_{1}+\sqrt{\frac{m_{\mathrm{H}}}{m_{\mathrm{D}}}}\left(\mathbf{r}_{1}^{(k)}-\overline{\mathbf{r}}_{1}\right),
$$

where $\overline{\mathbf{r}}_{i}$ represents the centroid of the quantum polymer associated to the $i$ th particle, namely,

$$
\overline{\mathbf{r}}_{i}=\frac{1}{P} \sum_{k=1}^{P} \mathbf{r}_{i}^{(k)}
$$

The ensemble averages that appear in the numerator and denominator in Eq. (9) represent conditional averages of the type $^{33}$

$$
\langle\mathcal{O}\rangle_{\mathcal{A}, m_{\mathrm{H}}}=\frac{\left\langle\frac{1}{P} \sum_{k=1}^{P} \eta_{\mathcal{A}}\left(\mathbf{r}_{1}^{(k)}\right) \mathcal{O}\right\rangle_{m_{\mathrm{H}}}}{\left\langle\frac{1}{P} \sum_{k=1}^{P} \eta_{\mathcal{A}}\left(\mathbf{r}_{1}^{(k)}\right)\right\rangle_{m_{\mathrm{H}}}}
$$

which are harvested with the masses of the light particles set to $m_{\mathrm{H}}$. The characteristic function $\eta_{\mathcal{A}}\left(\mathbf{r}_{1}^{(k)}\right)$ enforces boundaries for configuration subspaces: $\eta_{\mathcal{A}}\left(\mathbf{r}_{1}^{(k)}\right)$ is unity if, at the $k$-th imaginary time slice, the tagged particle 1 acts as $\mathrm{H}$-bonddonor to an $\mathcal{A}$-species $(\mathcal{A}=\mathrm{W}, \mathrm{X})$ and is zero otherwise. Incidentally, we remark that, due to cancellation effects, we found no meaningful differences between the results obtained from Eq. (13) and those obtained using boundary conditions imposed in terms of the corresponding centroid coordinate, namely,

$$
\langle\mathcal{O}\rangle_{\mathcal{A}, m_{\mathrm{H}}} \sim \frac{\left\langle\eta_{\mathcal{A}}\left(\overline{\mathbf{r}}_{1}\right) \mathcal{O}\right\rangle_{m_{\mathrm{H}}}}{\left\langle\eta_{\mathcal{A}}\left(\overline{\mathbf{r}}_{1}\right)\right\rangle_{m_{\mathrm{H}}}} .
$$

The two estimators in Eq. (9) were computed on the fly, along a single simulation experiment, in which all molecules correspond to $\mathrm{H}_{2} \mathrm{O}$.

Results for fractionation ratios for aqueous solutions at ambient conditions are listed in the second column of Table I. Clearly, the result for $\mathrm{F}^{-}$contrasts with the rest of the halides and reveal that the HOD $\cdots \mathrm{F}^{-}$coordination is more stable than the alternative, $\mathrm{DOH} \cdots \mathrm{F}^{-}$pattern. In the rest of the series, the relative stability reverses; this feature becomes more marked, the larger the size of the ion considered.

The comparison between simulation predictions and experimental results ${ }^{34,35}$ is instructive. In this case, the experimental results for the relative isotopic stabilities in aqueous electrolytes were estimated from indirect methods that involve measuring $\mathrm{D} / \mathrm{H}$ ratios in aqueous gas phases in equilibrium with the solutions. The interpretation of experimental data was based on a simplified, two state model for the water molecules in the solution: on the one hand, free states $\left[\mathrm{H}_{2} \mathrm{O}(\mathrm{f})\right]$, with similar characteristics to those prevailing in pure water phases and, on the other, bound states $\left[\mathrm{H}_{2} \mathrm{O}(\mathrm{b})\right]$, corresponding to molecules lying in the first solvation shell of the ions. The isotopic exchange equilibrium is expressed in terms of the following expression

$$
\mathrm{H}_{2} \mathrm{O}(\mathrm{f})+\mathrm{HDO}(\mathrm{b}) \leftrightarrow \mathrm{H}_{2} \mathrm{O}(\mathrm{b})+\mathrm{HDO}(\mathrm{f}),
$$

where no distinction was made whether the articulation between the HDO(b) molecule and the solute ionic species is established via the $\mathrm{H}$ or D donor-isotope. Still, the translocation described in the previous two-state equation would be equivalent to that described in Eq. (8) if the local characteristics of the hydrogen bonds connecting the first and second solvation shells of the solutes were similar to those prevailing in the bulk. As we will show in what follows, such hypothesis is found to be valid for the simple anionic species investigated here.

Experimental information reported in Ref. 34 is listed in column three of Table I; the dispersions in the entries reflect effects derived from the presence of different counterions, which we did not explore in the present study. Note that our predictions capture the correct order of magnitude of the isotope effects and reproduce the experimental size trend along the series of halides. Still, the simulation result for $\Delta A$ for the $\mathrm{I}^{-}$case looks overestimated compared with the corresponding entry in column 3 . As a possible explanation to account for this discrepancy, one could reasonably speculate that the accuracy of our simple ion-water interaction might not be sufficient to reproduce the experimental results, most notably

TABLE I. Free energy difference for the isotopic exchange reaction shown in Eq. (8).

\begin{tabular}{lccccc}
\hline & \multicolumn{2}{c}{$-10^{3} \ln \alpha^{\mathrm{a}}$} & & \multicolumn{2}{c}{$\Delta A^{\mathrm{a}}(\mathrm{meV})$} \\
\cline { 2 - 3 } \cline { 5 - 6 } Ion & Equation (9) & Reference 34 & & Equation (9) & Equation (17) \\
\hline $\mathrm{F}^{-}$ & $-46 \pm 2$ & & & $-1.18 \pm 0.05$ & $-1.46 \pm 0.06$ \\
$\mathrm{Cl}^{-}$ & $6 \pm 2$ & $6-14$ & & $0.15 \pm 0.05$ & $0.12 \pm 0.06$ \\
$\mathrm{Br}^{-}$ & $17 \pm 2$ & $13-16$ & & $0.44 \pm 0.05$ & $0.41 \pm 0.06$ \\
$\mathrm{I}^{-}$ & $26 \pm 2$ & $18-19$ & & $0.67 \pm 0.05$ & $0.76 \pm 0.06$ \\
\hline \hline
\end{tabular}

${ }^{\mathrm{a}}$ The uncertainties correspond to standard deviations from three statistically independent, 1 ns simulation runs. 
due to the absence of explicit polarization contributions. It is well documented that the incorporation of the latter electronic fluctuations may lead to important modifications in classical simulation predictions of ionic solvation, most notably in the case of large anions. ${ }^{36,37}$ Unfortunately, we failed to find information for $\alpha$ for the singular case of $\mathrm{F}^{-}$. However, and in order to support the qualitative change in the isotopic stability that we obtained from our simulations, one can bring into consideration electrochemical cell measurements ${ }^{34,38}$ that show that $\mathrm{LiF}, \mathrm{NaF}$, and $\mathrm{KF}$ exhibit more positive standard free energies in $\mathrm{H}_{2} \mathrm{O}$ than in $\mathrm{D}_{2} \mathrm{O}$. Although this thermodynamic information is not transferable here in a direct fashion, it is consistent with a gain in stability in passing from $\mathrm{OH} \cdots \mathrm{F}^{-}$to $\mathrm{OD} \cdots \mathrm{F}^{-}$ coordination.

A standard thermodynamic integration treatment for $\Delta A$ reveals that the latter quantity can be computed from differences in $\langle T\rangle$, the average quantum kinetic energies of the tagged atoms with different coordinations, namely, ${ }^{39}$

$$
\Delta A=\int_{m_{\mathrm{H}}}^{m_{\mathrm{D}}} \frac{\langle T\rangle_{\mathrm{W}, \mu}}{\mu}-\frac{\langle T\rangle_{\mathrm{X}, \mu}}{\mu} \mathrm{d} \mu .
$$

The values of $\langle T\rangle_{\mathcal{A}, \mu}$ are themselves reflective of complex interplays controlled by the local characteristics of intra and intermolecular potential energy fields acting on the tagged particles. A more clear interpretation of the latter feature can be obtained by considering the following harmonic approximation for the free energy difference $e^{40}$

$$
\Delta A \sim 2\left[1-\left(m_{\mathrm{H}} / m_{\mathrm{D}}\right)^{1 / 2}\right]\left(\langle T\rangle_{\mathrm{W}, m_{\mathrm{H}}}-\langle T\rangle_{\mathrm{X}, m_{\mathrm{H}}}\right),
$$

which can be obtained from Eq. (16), assuming that $\langle T\rangle_{\mathcal{A}, \mu}$ $\propto \mu^{-1 / 2}$ along the mass transformation path. This approximate expression is particularly revealing, since it clearly indicates that $\Delta A$ is the result of a competition between the values of the kinetic energies of the light $\mathrm{H}$-isotope at ionic and water connective positions.

Estimates for $\Delta A$ evaluated from Eqs. (9) and (17) are listed in the last two columns of Table I and plotted in Fig. 2 where we also have plotted direct experimental information. For the harmonic approximation [Eq. (17)], the values for $\langle T\rangle_{\mathcal{A}, m_{\mathrm{H}}}$ were obtained from the virial estimate reported in Ref. 41. The general agreement looks satisfactory, with perhaps less accuracy for the smallest ion. As possible explanations for the observed discrepancies, one can bring into consideration the highly anharmonic nature of the effective stretching

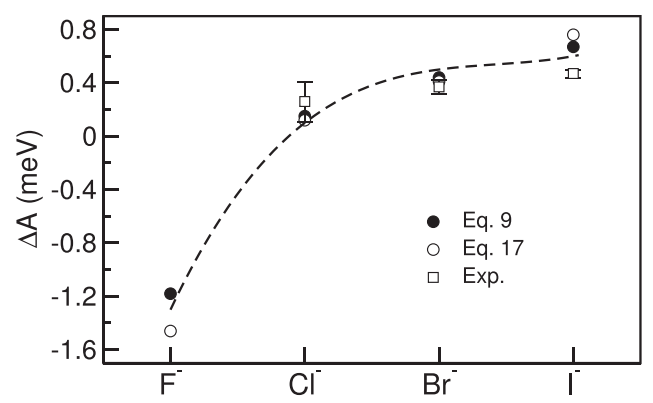

FIG. 2. Free energy difference for the isotope exchange reaction shown in Eq. (8). Results from Eqs. (9) and (17) are shown with filled and open circles, respectively; open squares correspond to experimental results from Ref. 34. The curve is a guide to the eye. potential of the proton along the $\mathrm{X}^{-}-\mathrm{H}-\mathrm{O}$ direction ${ }^{42,43}$ as a result of the strong Coulomb coupling between the proton and the nearby $\mathrm{F}^{-}$. Note that, as expected, an increment in $\langle T\rangle_{\mathrm{X}, \mathrm{m}_{\mathrm{H}}}$ in Eq. (17) brings the estimate for $\Delta A$ more negative. For the purposes of the present discussion, modifications in ionsolvent potentials are not needed; the quality of the agreement achieved indicates that our approximate treatment of interparticle interactions is sufficient to capture the phenomenon along the complete series of simple halides. Incidentally, we remark that the inversion in the relative stabilities of $\mathrm{X}^{-} \cdots \mathrm{DOH}$ versus $\mathrm{X}^{-} \cdots \mathrm{HOD}$ connectivity patterns in passing from $\mathrm{F}^{-}$to larger anions, looks similar to the one observed along $\mathrm{HBs}$ in $\mathrm{XH} \cdots \mathrm{OH}_{2}$ and $\mathrm{X}^{-} \cdots \mathrm{H}^{+}\left(\mathrm{H}_{2} \mathrm{O}\right)$ moieties embedded in water clusters at $T=25 \mathrm{~K} .{ }^{11}$ However, in the present case, the magnitude of the nuclear quantum stabilization, expressed in terms of $\beta \Delta A$, is, at least, two orders of magnitude smaller than the one observed at cryogenic temperatures.

The consideration of a geometrical description introduces a new opportunity to rationalize these tendencies. Following previous analysis, ${ }^{21,22,44}$ one can decompose the values of the average kinetic energies into three relevant orthogonal projections: (i) $\left\langle T_{\|}\right\rangle$corresponds to a direction parallel to the $\mathrm{O}-\mathrm{H}$ vector participating in a tagged $\mathrm{H}$-bond; (ii) $\left\langle T_{i p}\right\rangle$ corresponds to a direction in the molecular plane, perpendicular to the $\mathrm{O}-\mathrm{H}$ vector; (iii) $\left\langle T_{o p}\right\rangle$ corresponds to an out-of-plane direction, perpendicular to the plane of the molecule.

Results for $\langle T\rangle_{\mathcal{A}, m_{\mathrm{H}}}$ are depicted in Fig. 3 and listed in Table II where we have also included results for the corresponding projections. The inspection of the entries immediately reveals the following features: (i) the data for $\langle T\rangle_{\mathrm{X}, m_{\mathrm{H}}}$ listed in column 2 and shown in blue in the top-left panel of Fig. 3 exhibits a gradual decrease as the size of the halide increases; (ii) the trend in the entries of $\left\langle T_{\|}\right\rangle_{\mathrm{X}, m_{\mathrm{H}}}$ (column 3 ; top-right panel, blue) is the opposite, showing a steady increase as the basicity of the ion decreases; (iii) combined contributions from the in-plane and out-of-plane, perpendicular projections (columns 4 and 5; bottom panels, blue) show the opposite trend and overwhelm the parallel contributions; and (iv) the entries in the last columns on the right hand side (red bars in Fig. 3) reveal that the energetics along W$\mathrm{W}$ H-bonds linking the first and second solvation shells, in fact, remain virtually unchanged in all four cases and virtually identical to the values for bulk water (last row). These results might not be expected, considering the large solvent reorganization energies normally involved in the solvation of simple ionic species, ${ }^{4}$ but are consistent with the hypothesis leading to the equivalence between the expressions shown in Eqs. (8) and (15). As such, the general picture that emerges from this set of data indicates that the overall differences between $\langle T\rangle_{\mathrm{X}, m_{\mathrm{H}}}$ and $\langle T\rangle_{\mathrm{W}, m_{\mathrm{H}}}$ - and consequently, the resulting values of $\triangle A$ - are dominated by contributions from directions perpendicular to the relevant $\mathrm{O}-\mathrm{H}$ direction along ionic $\mathrm{H}$-bonds. This direct observation is consistent with conclusions reported in previous analyses, ${ }^{12,21,22,44}$ but contrasts with what is found in the aforementioned HX solvation scenarios, where stability control is transferred to differences in parallel projections. $^{11}$

Physical interpretations of the last features are afforded by basic quantum arguments that relate average kinetic 


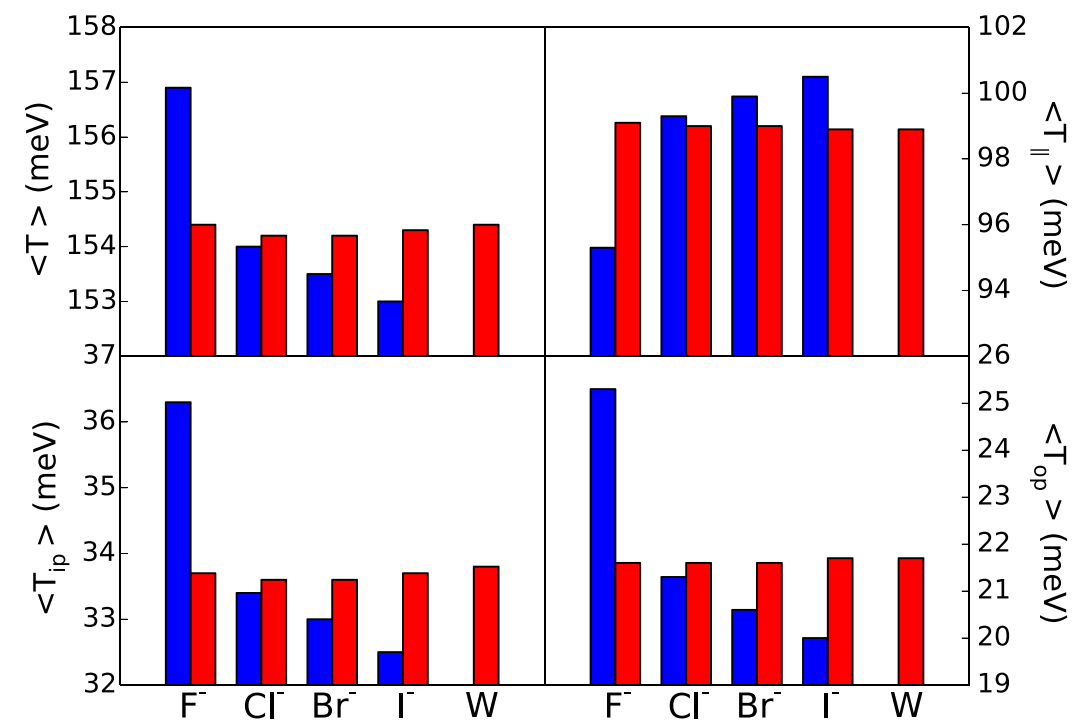

FIG. 3. Quantum kinetic energies for $\mathrm{H}$-atoms in the first solvation shell of different $\mathrm{X}^{-}$halides obtained from the virial estimator (see text). $\mathrm{O}-\mathrm{H} \cdots \mathrm{X}^{-}$connetivity (blue); $\mathrm{O}-\mathrm{H} \cdots \mathrm{O}$ connectiviy (red). We also included results for bulk water $(\mathrm{W})$. energies and the extent of the quantum spatial delocalization of the particles. As the size of the halide solute increases, the solute-solvent Coulomb coupling decreases, leading to increasing curvature of the net potential along the intramolecular $\mathrm{O}-\mathrm{H}$ stretch, moving toward a free $\mathrm{OH}$ and increasing the parallel contributions to the quantum kinetic energy. The description along perpendicular directions contrasts sharply. First, note that the values of $\left\langle T_{i p}\right\rangle$ and $\left\langle T_{o p}\right\rangle$ are typically between three and four times smaller that $\left\langle T_{\|}\right\rangle$, revealing that they can be ascribed to much softer, libration-like motions of molecules located in the immediate halide solvation shell. Under these circumstances, the presence of weaker solutesolvent Coulomb couplings leads to gradual reductions in the directionality of the ionic $\mathrm{H}$-bond and to increasing spatial delocalization of the quantum protons in these perpendicular directions. The entries in Table II would also indicate that, along the $\mathrm{Cl}^{-}, \mathrm{Br}^{-}, \mathrm{I}^{-}$sequence, the degree of directional confinement along perpendicular directions is less marked than the one registered along a typical $\mathrm{OH} \cdots \mathrm{O}$ bond in bulk water. For the coordination with $\mathrm{F}^{-}$, the scenario changes and the corresponding perpendicular contribution surpasses the one registered in $\mathrm{W}-\mathrm{W}$ H-bonds. The physical picture that emerges would indicate that this delicate balance between competing parallel and perpendicular components of the kinetic energy represents the key element controlling the isotopic preferential solvation of ionic species. We note that a similar reasoning also explains the propensity registered in the connectivity of the simplest $\mathrm{I}^{-}$. DOH dimer, where the interplay is between ionic-bonded and dangling positions. ${ }^{12,45}$
The previous characteristics can also be, in part, inferred from the analysis of $\operatorname{IR}^{9,10}$ and Raman ${ }^{7,46}$ signals. Within the spirit of the RPMD approximation, the vibrational spectrum $I(\omega)$ can be cast in terms of the Fourier transform of the second derivative of the Kubo-transformed, RPMD time correlation function of the dipole moment, namely, ${ }^{15,19}$

$$
I(\omega) \propto \int_{0}^{\infty} C_{\dot{\mu} \dot{\mu}}(t) \cos (\omega t) \mathrm{d} t,
$$

where

$$
C_{\dot{\mu} \dot{\mu}}(t)=\langle\dot{\mu}(t) \cdot \dot{\mu}(0)\rangle_{\mathrm{RPMD}}
$$

and

$$
\dot{\boldsymbol{\mu}}(t)=\frac{1}{P} \sum_{k=1}^{P}\left[\sum_{j=1}^{3 N_{w}} z_{j} e \mathbf{v}_{j}^{(k)}(t)\right] .
$$

In principle, in Eq. (20), the sum over the index $j$ involves $3 N_{w}$ sites, with partial charges $z_{j} e$ and velocities $\mathbf{v}_{j}^{(k)}$ evaluated at the $k$-th imaginary time slice. In the present case, however, we restricted the sampling of the correlation function shown in Eq. (19) to molecules lying at the ionic first solvation shell. In order to avoid the presence of spurious frequencies originating from intra-polymer modes, ${ }^{47}$ time correlation functions were computed from separate simulations using the Thermostatted Ring Polymer Molecular Dynamics (TRPMD) method. ${ }^{48}$ This procedure requires the decoupling of the Langevin thermostat from the modes associated to the centroids of the isomorphic polymers.

In order to establish a correspondence between experimental and simulation results, we found it instructive to

TABLE II. Average quantum kinetic energies, with parallel and perpendicular contributions, for $\mathrm{H}$ atoms participating different hydrogen-bonds. ${ }^{\text {a }}$

\begin{tabular}{lcccccccc}
\hline \hline Ion & $\langle T\rangle_{\mathrm{X}, m_{\mathrm{H}}}$ & $\left\langle T_{\|}\right\rangle_{\mathrm{X}, m_{\mathrm{H}}}$ & $\left\langle T_{\mathrm{ip}}\right\rangle_{\mathrm{X}, m_{\mathrm{H}}}$ & $\left\langle T_{\mathrm{op}}\right\rangle_{\mathrm{X}, m_{\mathrm{H}}}$ & $\langle T\rangle_{\mathrm{W}, m_{\mathrm{H}}}$ & $\left\langle T_{\|}\right\rangle_{\mathrm{W}, m_{\mathrm{H}}}$ & $\left\langle T_{\mathrm{ip}}\right\rangle_{\mathrm{W}, m_{\mathrm{H}}}$ & $\left\langle T_{\mathrm{op}}\right\rangle_{\mathrm{W}, m_{\mathrm{H}}}$ \\
\hline $\mathrm{F}^{-}$ & 156.9 & 95.3 & 36.3 & 25.3 & 154.4 & 99.1 & 33.7 & 21.6 \\
$\mathrm{Cl}^{-}$ & 154.0 & 99.3 & 33.4 & 21.3 & 154.2 & 99.0 & 33.6 & 21.6 \\
$\mathrm{Br}^{-}$ & 153.5 & 99.9 & 33.0 & 20.6 & 154.2 & 99.0 & 33.6 & 21.6 \\
$\mathrm{I}^{-}$ & 153.0 & 100.5 & 32.5 & 20.0 & 154.3 & 98.9 & 33.7 & 21.7 \\
Bulk water & & & & & 154.4 & 98.9 & 33.8 & 21.7 \\
\hline \hline
\end{tabular}

anergies are expressed in meV. Uncertainties in the entries are of the order of $\sim 0.1 \mathrm{meV}$. 
concentrate on the behavior of an auxiliary system, comprising an infinitely dilute anion in a solvent composed of HOD molecules exclusively. In doing so, signals corresponding to stretching modes in molecules participating in HOD $\cdots \mathrm{X}^{-}$ and in DOH $\cdots X^{-}$motifs are clearly distinguished. This simplification, in turn, avoids the consideration of overlapping signals from collective stretching motions than would otherwise prevail in a more realistic simulation with mixed solvents, combining $\mathrm{H}_{2} \mathrm{O}$ or $\mathrm{D}_{2} \mathrm{O}$ as major components.

In the top panel of Fig. 4, we present results for the high frequency IR bands obtained from HOD molecules lying at the ionic first solvation shell (open circles). The overall absorption lineshapes show two broad sub-bands at $\omega \sim 3300-3500 \mathrm{~cm}^{-1}$ and $\omega \sim 2450-2600 \mathrm{~cm}^{-1}$, corresponding to localized stretching motions involving $\mathrm{H}$ and $\mathrm{D}$ isotopic species, respectively. For the $\mathrm{F}^{-}$case, the overall positions of both sub-bands are clearly red-shifted with respect to the corresponding $\mathrm{I}^{-}$signals, in accord with the stronger nature of the ionic H-bonds found for smaller ions. The decomposition of the IR signals into contributions from molecules with different ionic connectivities provides additional details. For the $\mathrm{F}^{-}$case, the highest frequency of the $\mathrm{OH}$ sub-band at $\omega \sim 3490 \mathrm{~cm}^{-1}$ (dotted line) includes contributions from $\mathrm{H}$ motions localized along $\mathrm{OH} \cdots \mathrm{O}$ bonds, linking the first and second solvation shells. Note that this frequency regime practically coincides with the predicted $\mathrm{O}-\mathrm{H}$ stretch signal in the neat auxiliary HOD solvent (solid line), $\sim 100 \mathrm{~cm}^{-1}$ blue-shifted with respect to the experimental result ${ }^{10}$ for a $1 \mathrm{M}$ solution of $\mathrm{HDO}$ in $\mathrm{D}_{2} \mathrm{O}$ (solid arrow). Contributions from modes along ionic $\mathrm{H}$-bonds are predominant in the low frequency flank of the $\mathrm{H}$ sub-band, located at

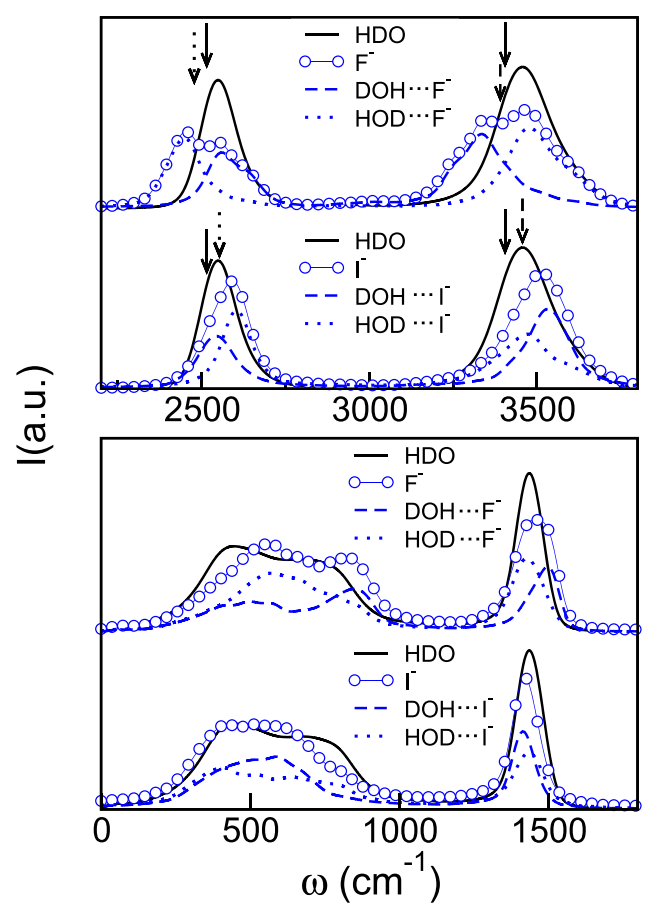

FIG. 4. Infrared spectra of aqueous systems [Eq. (18)]. Top panel: Stretching bands for HOD in the first solvation shells of $\mathrm{F}^{-}$and of $\mathrm{I}^{-}$(blue open circles) and their partial contributions from molecules with different ionic-water connectivities (dashed and dotted lines). Solid lines correspond to results for the neat HDO. The arrows indicate the positions of the centers of the experimental bands (see text). Bottom panel: same as top, but for the low frequency part of the IR spectrum. $\omega \sim 3330 \mathrm{~cm}^{-1}$ (dashed line) and correlate with the position of the experimental $\mathrm{OH}$ stretching band in a $6 \mathrm{M}$ solution of $\mathrm{KF}$ in $\mathrm{H}_{2} \mathrm{O}$ (dashed arrow). ${ }^{10}$ The inspection of the O-D subband reveals results that mirror images of those in the $\mathrm{O}-\mathrm{H}$ sub-band. More importantly, the predicted trend in the peak positions agrees with that reported in Ref. 9 for the OD $\cdots \mathrm{O}$ stretching band for a $4 \mathrm{wt}$ \% $\mathrm{D}_{2} \mathrm{O}$ in $\mathrm{H}_{2} \mathrm{O}$ mixture (solid arrow) and for the $\mathrm{OD} \cdots \mathrm{F}^{-}$stretching band for $\mathrm{KF} / \mathrm{D}_{2} \mathrm{O} / \mathrm{H}_{2} \mathrm{O}$ ternary mixture (dotted arrow).

For the $\mathrm{I}^{-}$case, the positions of the signals for $\mathrm{W}-\mathrm{W}$ and $\mathrm{X}^{-}-\mathrm{W}$ bonding interchange; the highest frequency contributions correspond to modes localized along $\mathrm{OH} \cdots \mathrm{I}^{-}$bonds, which appear at $\Delta \omega \sim 100 \mathrm{~cm}^{-1}$, blue-shifted with respect to the neat solvent signal. In this case, the positioning of the four $\mathrm{H}$ and $\mathrm{D}$ sub-bands agrees with experimental results ${ }^{9,10}$ indicated by arrows.

The bottom panel of Fig. 4 contains results for the low frequency bands of the spectra that correspond to bending and intermolecular (librational) modes. The characterization of the latter sub-band is not simple since it involves a variety of collective motions involving librational and hindered translational modes. ${ }^{49}$ Nevertheless, one can still clearly perceive that the overall trends in the frequency shifts are opposite to those observed for the stretching modes. Therefore, the simple interpretation, based on establishing a direct correspondence between (i) stretching modes and parallel projections of the quantum kinetic energies and (ii) bending/librational modes and perpendicular projections, corroborates our earlier conclusions concerning the origins of the isotopic preferential solvation of simple ionic species.

\section{CONCLUDING REMARKS}

Summarizing, in this paper, we have presented pathintegral-molecular-dynamics results that shed light on the microscopic origins of the relative thermodynamic stabilizations of $\mathrm{OH} \cdots \mathrm{X}^{-}$and $\mathrm{OD} \cdots \mathrm{X}^{-}$coordination along a series of simple halides in aqueous solutions at ambient conditions. The present results complement previous ones reported for aqueous nanoclusters at low temperatures, expanding the thermal interval and providing a more comprehensive description of different halides in solutions. Our results corroborate experimental thermodynamic results indicating that, for large anions, the $\mathrm{OH} \cdots \mathrm{X}^{-}$motif is the most stable articulation. This characteristic reverses for the $\mathrm{F}^{-}$case, where coordination via a deuterated bond is thermodynamically preferred. Moreover, these tendencies are the result of a balance between the contributions to the potential energy surface describing the forces on the light atoms along ion-water and water-water bonds. From a quantum perspective, these geometric characteristics are clearly reflected in the proton delocalization and in the decomposition of the corresponding kinetic energies along perpendicular and parallel directions with respect to the linear intermolecular bond. Similarly to what was reported for "dangling" versus "connective" positions at liquid/air interfaces at ambient conditions, ${ }^{44}$ the thermodynamic stabilities of the different motifs are controlled by perpendicular projections of the kinetic energy. This contrasts with the results from $\mathrm{HX}\left(\mathrm{H}_{2} \mathrm{O}\right)_{n}$ clusters at low temperatures, where differences in the 
parallel components prevail. Consistently, the latter values clearly correlate with simulated spectral shifts in infrared spectra; the latter, in turn, agree with those registered in direct experimental measurements, bringing additional support to the quality of our simulation predictions.

\section{ACKNOWLEDGMENTS}

D.L. is a staff member of CONICET-Argentina. P.J.R. acknowledges the support of the U.S. National Science Foundation (No. CHE-1362381). The research work of D.L. is partially funded by CONICET-Argentina (PIP 11220110100464).

${ }^{1}$ W. H. Robertson and M. A. Johnson, Annu. Rev. Phys. Chem. 54, 173 (2003).

${ }^{2}$ R. W. Gurney, Ionic Processes in Solution (McGraw-Hill, New York, 1953).

${ }^{3}$ K. D. Collins and M. W. Washabaugh, Q. Rev. Biophys. 18, 323 (1985).

${ }^{4}$ Y. Marcus, Chem. Rev. 109, 1346 (2009).

${ }^{5}$ M. F. Kropman and H. J. Bakker, Science 291, 2118 (2001).

${ }^{6}$ A. W. Omta, M. F. Kropman, S. Wourtersen, and H. J. Bakker, Science 301, 347 (2003).

${ }^{7}$ J. D. Smith, R. J. Saykally, and P. L. Geissler, J. Am. Chem. Soc. 129, 13847 (2007).

${ }^{8}$ P. Ball and J. E. Hallsworth, Phys. Chem. Chem. Phys. 17, 8297 (2015).

${ }^{9}$ Z. S. Nickolov and J. D. Miller, J. Colloid Interface Sci. 287, 572 (2005).

${ }^{10}$ M. F. Kropman and H. J. Bakker, Chem. Phys. Lett. 370, 741 (2003).

${ }^{11}$ Y. E. Litman, P. E. Videla, J. Rodriguez, and D. Laria, J. Phys. Chem. A 120, 7213 (2016).

${ }^{12}$ P. E. Videla, P. J. Rossky, and D. Laria, J. Phys. Chem. B 119, 11783 (2015).

${ }^{13}$ S. Habershon, Phys. Chem. Chem. Phys. 16, 9154 (2014).

${ }^{14}$ D. Chandler and P. Wolynes, J. Chem. Phys. 74, 4078 (1981).

${ }^{15}$ S. Habershon, G. S. Fanourgakis, and D. E. Manolopoulos, J. Chem. Phys. 129, 074501 (2008).

${ }^{16}$ M. Tuckerman, B. J. Berne, and G. J. Martyna, J. Chem. Phys. 97, 1990 (1992).

${ }^{17}$ M. Ceriotti, M. Parrinello, T. E. Markland, and D. E. Manolopoulos, J. Chem. Phys. 133, 124104 (2010).

${ }^{18}$ U. Essmann et al., J. Chem. Phys. 103, 8577 (1995).
${ }^{19}$ S. Habershon, T. E. Markland, and D. E. Manolopoulos, J. Chem. Phys. 131, 024501 (2009).

${ }^{20}$ J. L. F. Abascal and C. Vega, J. Chem. Phys. 123, 234505 (2005).

${ }^{21}$ T. E. Markland and B. J. Berne, Proc. Natl. Acad. Sci. U. S. A. 109, 7988 (2012).

${ }^{22}$ J. Liu et al., J. Phys. Chem. C 117, 2944 (2013).

${ }^{23}$ T. Peng, T. Chang, X. Sun, A. V. Nguyen, and L. X. Dang, J. Mol. Liq. 173, 47 (2012).

${ }^{24}$ S. Joung and T. E. Cheatham III, J. Phys. Chem. B 112, 9020 (2008).

${ }^{25}$ K. P. Jensen and W. L. Jorgensen, J. Chem. Theory Comput. 2, 1499 (2006).

${ }^{26}$ G. Lamoureux and B. Roux, J. Phys. Chem. B 110, 3308 (2006).

${ }^{27} \mathrm{H}$. Yu et al., J. Chem. Theory Comput. 6, 774 (2010).

${ }^{28}$ A. Karmakar and A. Chandra, J. Phys. Chem. B 119, 8561 (2015).

${ }^{29}$ A. Luzar and D. Chandler, Phys. Rev. Lett. 76, 928 (1996).

${ }^{30}$ A. Chandra, J. Phys. Chem. B 107, 3899 (2003).

${ }^{31}$ I. R. Craig and D. E. Manolopoulos, J. Chem. Phys. 121, 3368 (2004).

${ }^{32}$ S. Habershon, D. E. Manolopoulos, T. E. Markland, and T. F. Miller III, Annu. Rev. Phys. Chem. 64, 387 (2013).

${ }^{33}$ B. Cheng and M. Ceriotti, J. Chem. Phys. 141, 244112 (2014).

${ }^{34}$ M. Kakiuchi, Z. Naturforsch. 52a, 811 (1997).

${ }^{35}$ M. Kakiuchi, Z. Naturforsch. 62a, 721 (2007).

${ }^{36}$ P. Jungwirth and D. J. Tobias, J. Phys. Chem. B 106, 6361 (2002).

${ }^{37}$ P. Jungwirth and D. J. Tobias, J. Phys. Chem. A 106, 379 (2002).

${ }^{38}$ J. Greyson, J. Phys. Chem. 71, 2210 (1967).

${ }^{39}$ J. Vanicek and W. H. Miller, J. Chem. Phys. 127, 114309 (2007).

${ }^{40}$ M. Ceriotti and T. E. Markland, J. Chem. Phys. 138, 14112 (2013).

${ }^{41}$ M. F. Herman, J. Bruskin, and B. J. Berne, J. Chem. Phys. 76, 5150 (1982).

${ }^{42}$ B. F. Yates, H. F. Shaefer III, T. J. Lee, and J. E. Rice, J. Am. Chem. Soc. 110, 6237 (1988).

${ }^{43}$ W. H. Thompson and J. T. Hynes, J. Am. Chem. Soc. 122, 6278 (2000).

${ }^{44}$ D. M. Wilkins, D. E. Manolopoulos, and L. X. Dang, J. Chem. Phys. 142, 064509 (2015).

${ }^{45}$ E. G. Diken, J.-W. Shin, E. A. Price, and M. A. Johnson, Chem. Phys. Lett. 387, 17 (2004).

${ }^{46}$ G. E. Walrafen, J. Chem. Phys. 122, 094510 (2005).

${ }^{47}$ A. Witt, S. D. Ivanov, M. Shiga, H. Forbert, and D. Marx, J. Chem. Phys. 130, 194510 (2009).

${ }^{48}$ M. Rossi, M. Ceriotti, and D. E. Manolopoulos, J. Chem. Phys. 140, 234116 (2014).

${ }^{49}$ I. Nakagawa and T. Shimanouchi, Spectrochim. Acta 20, 429 (1964). 